\title{
A New Quintic Spline Method for Integro Interpolation and Its Error Analysis
}

\section{Feng-Gong Lang}

School of Mathematical Sciences, Ocean University of China, Qingdao 266100, China; fenggonglang@ouc.edu.cn; Tel.: +86-532-6678-7153

Academic Editor: Alicia Cordero

Received: 24 December 2016; Accepted: 1 March 2017; Published: 3 March 2017

\begin{abstract}
In this paper, to overcome the innate drawbacks of some old methods, we present a new quintic spline method for integro interpolation. The method is free of any exact end conditions, and it can reconstruct a function and its first order to fifth order derivatives with high accuracy by only using the given integral values of the original function. The approximation properties of the obtained integro quintic spline are well studied and examined. The theoretical analysis and the numerical tests show that the new method is very effective for integro interpolation.
\end{abstract}

Keywords: quintic spline; integral value; integro interpolation; artificial end condition; error analysis

\section{Introduction}

Assume that $y=y(x)$ is an unknown univariate real-valued function over $[a, b]$. Let:

$$
\Delta:=\left\{a=x_{0}<x_{1}<\cdots<x_{n}=b\right\}
$$

be the uniform partition of $[a, b]$ with step length $h:=\frac{b-a}{n}$, and let:

$$
I_{j}:=\int_{x_{j}}^{x_{j+1}} y(x) d x \quad(j=0,1, \ldots, n-1)
$$

be the known integral values of $y=y(x)$ over the subintervals.

The interpolation function $p=p(x)$ that satisfies:

$$
\int_{x_{j}}^{x_{j+1}} p(x) d x=I_{j} \quad(j=0,1, \ldots, n-1)
$$

is called integro interpolation. The problem arises in many fields, such as numerical analysis, mathematical statistics, environmental science, mechanics, electricity, climatology, oceanography, and so on. We refer to [1-19] for its applied backgrounds and some recent developments.

In this paper, we will mainly focus on the quintic spline methods; see $[2,14,17-19]$ for the existing ones.

The method in [2] was based on the quintic Hermite-Birkhoff polynomials. The method was very complicated because it mainly required solving two linear systems. Furthermore, besides the integral values (2), the method must use seven additional exact end conditions in terms of $y\left(x_{0}\right), y^{\prime}\left(x_{0}\right)$, $y^{\prime}\left(x_{1}\right), y^{\prime}\left(x_{n-1}\right), y^{\prime}\left(x_{n}\right), y^{\prime \prime \prime}\left(x_{0}\right)$ and $y^{\prime \prime \prime}\left(x_{n}\right)$. Later, a new algorithm was given in [18] to simplify the construction of integro quintic spline. It mainly required solving two linear three-diagonal systems. It was kind of simpler than that of [2]. However, the algorithm needed five special and proper exact end conditions in terms of $y\left(x_{0}\right), y^{\prime}\left(x_{1}\right), y^{\prime}\left(x_{n-1}\right), y^{\prime \prime \prime}\left(x_{1}\right)$ and $y^{\prime \prime \prime}\left(x_{n-1}\right)$. The method in [14] was based on quintic B-splines. It was also very simple because it took advantage of the good properties of 
quintic B-splines. However, five additional exact end conditions in terms of $y\left(x_{0}\right), y\left(x_{1}\right), y\left(x_{2}\right), y\left(x_{n-1}\right)$ and $y\left(x_{n}\right)$ must be provided. In other words, these methods all need exact end conditions. This is an obvious drawback of them. New simple methods that are not dependent on exact end conditions are desired.

In [17], we have studied an effective method that was not dependent on any exact end conditions. We first obtained $n+1$ approximate function values at the knots and four approximate boundary derivative values from the integral values (2) and then used them to study a modified quintic spline interpolation problem. However, the method also had its own drawbacks. On the one hand, it needed $n+5$ artificial values, which brought higher computational cost; on the other hand, the obtained quintic spline did not agree with the given integral values (2) over the subintervals. In [19], a local integro quintic spline method was given. It was also not dependent on exact end conditions and was able to produce good approximations. However, the obtained local integro quintic spline also did not agree with the given integral values (2) over the subintervals. Hence, these methods also need improvements. New attempts on this problem are still necessary.

In this paper, we aim to develop a new effective method to overcome the above-mentioned drawbacks. We will first construct six artificial end conditions by using a similar technique to [17] and use them together with the integral values (2) to get a new kind of integro quintic spline; then, we will theoretically analyze and numerically examine the approximation properties of the new integro quintic spline. The new method is very effective, and it has the following advantages.

(I) The method is free of any exact end conditions, and it only requires five artificial end conditions, which can be easily obtained by simple computations from several integral values.

(II) The computational procedure of the method is concise and easy to implement.

(III) The obtained quintic spline agrees with the given integral values (2) over the subintervals.

(IV) The obtained quintic spline can provide satisfactory approximations to $y^{(k)}(x), k=0,1,2,3,4,5$.

Hence, this method is very applicable for the integro interpolation problem.

The remainder of this paper is organized as follows. In Section 2, we compute some artificial end conditions by using several integral values; in Section 3, we construct our new integro quintic spline with five artificial end conditions; the approximation abilities of the integro quintic spline are theoretically studied in Section 4 and numerically tested in Section 5; finally, we conclude our paper in Section 6.

\section{Artificial End Conditions}

In this section, we study some new artificial end conditions for integro interpolation.

It is assumed that $y=y(x)$ is a function of class $C^{7}[a, b]$ throughout this paper. In order to get the highest error orders, we will use seven boundary integral values to construct some proper linear combinations of them as the artificial end conditions. By expanding $y=y(x)$ at $x=x_{0}$ by using the Taylor formula and computing the integral on $\left[x_{0}, x_{m}\right], m=1,2, \ldots, 7$, we obtain:

$$
\begin{aligned}
\sum_{\ell=0}^{m-1} I_{\ell} & =\int_{x_{0}}^{x_{m}} y(x) d x \\
& =y_{0}(m h)+\frac{y_{0}^{\prime}}{2 !}(m h)^{2}+\frac{y_{0}^{\prime \prime}}{3 !}(m h)^{3}+\frac{y_{0}^{\prime \prime \prime}}{4 !}(m h)^{4} \\
& +\frac{y_{0}^{(4)}}{5 !}(m h)^{5}+\frac{y_{0}^{(5)}}{6 !}(m h)^{6}+\frac{y_{0}^{(6)}}{7 !}(m h)^{7}+O\left(h^{8}\right)
\end{aligned}
$$


For $\ell=1,2, \ldots, 7$, let $\lambda_{\ell}, \omega_{\ell}$ and $\mu_{\ell}$ be three parameters, such that:

$$
\left(\begin{array}{lllllll}
1 & 2 & 3 & 4 & 5 & 6 & 7 \\
1 & 2^{2} & 3^{2} & 4^{2} & 5^{2} & 6^{2} & 7^{2} \\
1 & 2^{3} & 3^{3} & 4^{3} & 5^{3} & 6^{3} & 7^{3} \\
1 & 2^{4} & 3^{4} & 4^{4} & 5^{4} & 6^{4} & 7^{4} \\
1 & 2^{5} & 3^{5} & 4^{5} & 5^{5} & 6^{5} & 7^{5} \\
1 & 2^{6} & 3^{6} & 4^{6} & 5^{6} & 6^{6} & 7^{6} \\
1 & 2^{7} & 3^{7} & 4^{7} & 5^{7} & 6^{7} & 7^{7}
\end{array}\right)\left(\begin{array}{lll}
\lambda_{1} & \omega_{1} & \mu_{1} \\
\lambda_{2} & \omega_{2} & \mu_{2} \\
\lambda_{3} & \omega_{3} & \mu_{3} \\
\lambda_{4} & \omega_{4} & \mu_{4} \\
\lambda_{5} & \omega_{5} & \mu_{5} \\
\lambda_{6} & \omega_{6} & \mu_{6} \\
\lambda_{7} & \omega_{7} & \mu_{7}
\end{array}\right)=\left(\begin{array}{lll}
1 & 0 & 0 \\
0 & 1 & 0 \\
0 & 0 & 1 \\
0 & 0 & 0 \\
0 & 0 & 0 \\
0 & 0 & 0 \\
0 & 0 & 0
\end{array}\right)
$$

Explicitly,

$$
\begin{aligned}
& \lambda_{1}=7, \quad \lambda_{2}=-\frac{21}{2}, \quad \lambda_{3}=\frac{35}{3}, \quad \lambda_{4}=-\frac{35}{4}, \quad \lambda_{5}=\frac{21}{5}, \quad \lambda_{6}=-\frac{7}{6}, \quad \lambda_{7}=\frac{1}{7}, \\
& \omega_{1}=-\frac{223}{20}, \quad \omega_{2}=\frac{879}{40}, \quad \omega_{3}=-\frac{949}{36}, \quad \omega_{4}=\frac{41}{2}, \quad \omega_{5}=-\frac{201}{20}, \quad \omega_{6}=\frac{1019}{360}, \quad \omega_{7}=-\frac{7}{20}, \\
& \mu_{1}=\frac{319}{45}, \quad \mu_{2}=-\frac{3929}{240}, \quad \mu_{3}=\frac{389}{18}, \quad \mu_{4}=-\frac{2545}{144}, \quad \mu_{5}=\frac{134}{15}, \quad \mu_{6}=-\frac{1849}{720}, \quad \mu_{7}=\frac{29}{90} \text {. }
\end{aligned}
$$

By using (4) and using these parameters $\lambda_{\ell}, \omega_{\ell}$ and $\mu_{\ell}, \ell=1,2, \ldots, 7$, as the linear combination coefficients, we obtain:

$$
\begin{aligned}
& \sum_{m=1}^{7}\left(\lambda_{m} \sum_{\ell=0}^{m-1} I_{\ell}\right) \\
= & \frac{1}{420}\left(1089 I_{0}-1851 I_{1}+2559 I_{2}-2341 I_{3}+1334 I_{4}-430 I_{5}+60 I_{6}\right) \\
= & y_{0} h+O\left(h^{8}\right), \\
& \sum_{m=1}^{7}\left(\omega_{m} \sum_{\ell=0}^{m-1} I_{\ell}\right) \\
= & \frac{1}{360}\left(-938 I_{0}+3076 I_{1}-4835 I_{2}+4655 I_{3}-2725 I_{4}+893 I_{5}-126 I_{6}\right) \\
= & \frac{1}{2} y_{0}^{\prime} h^{2}+O\left(h^{8}\right), \\
& \sum_{m=1}^{7}\left(\mu_{m} \sum_{\ell=0}^{m-1} I_{\ell}\right) \\
= & \frac{1}{720}\left(967 I_{0}-4137 I_{1}+7650 I_{2}-7910 I_{3}+4815 I_{4}-1617 I_{5}+232 I_{6}\right) \\
= & \frac{1}{6} y_{0}^{\prime \prime} h^{3}+O\left(h^{8}\right) .
\end{aligned}
$$

Similarly, we also can get some corresponding results at the right end point. Based on (5)-(7) and the corresponding results at the right end point, let:

$$
\begin{aligned}
& \widehat{y}_{0}=\frac{1}{420 h}\left(1089 I_{0}-1851 I_{1}+2559 I_{2}-2341 I_{3}+1334 I_{4}-430 I_{5}+60 I_{6}\right), \\
& \widehat{y}_{0}^{\prime}=\frac{1}{180 h^{2}}\left(-938 I_{0}+3076 I_{1}-4835 I_{2}+4655 I_{3}-2725 I_{4}+893 I_{5}-126 I_{6}\right), \\
& \widehat{y}_{0}^{\prime \prime}=\frac{1}{120 h^{3}}\left(967 I_{0}-4137 I_{1}+7650 I_{2}-7910 I_{3}+4815 I_{4}-1617 I_{5}+232 I_{6}\right),
\end{aligned}
$$


and:

$$
\begin{aligned}
& \widehat{y}_{n}=\frac{1}{420 h}\left(1089 I_{n-1}-1851 I_{n-2}+2559 I_{n-3}-2341 I_{n-4}+1334 I_{n-5}-430 I_{n-6}+60 I_{n-7}\right), \\
& \widehat{y}_{n}^{\prime}=\frac{1}{180 h^{2}}\left(938 I_{n-1}-3076 I_{n-2}+4835 I_{n-3}-4655 I_{n-4}+2725 I_{n-5}-893 I_{n-6}+126 I_{n-7}\right), \\
& \widehat{y}_{n}^{\prime \prime}=\frac{1}{120 h^{3}}\left(967 I_{n-1}-4137 I_{n-2}+7650 I_{n-3}-7910 I_{n-4}+4815 I_{n-5}-1617 I_{n-6}+232 I_{n-7}\right) .
\end{aligned}
$$

It is straightforward to prove that:

$$
\begin{array}{ll}
\widehat{y}_{0}-y_{0}=O\left(h^{7}\right), & \widehat{y}_{0}^{\prime}-y_{0}^{\prime}=O\left(h^{6}\right), \quad \widehat{y}_{0}^{\prime \prime}-y_{0}^{\prime \prime}=O\left(h^{5}\right), \\
\widehat{y}_{n}-y_{n}=O\left(h^{7}\right), & \widehat{y}_{n}^{\prime}-y_{n}^{\prime}=O\left(h^{6}\right), \quad \widehat{y}_{n}^{\prime \prime}-y_{n}^{\prime \prime}=O\left(h^{5}\right) .
\end{array}
$$

Let $\theta_{n}=\widehat{y}_{n}+\frac{1}{10} h^{2} \widehat{y}_{n}^{\prime \prime}$, by using (10) and (12); then, we get:

$$
\theta_{n}=\frac{1}{8400 h}\left(28549 I_{n-1}-65979 I_{n-2}+104730 I_{n-3}-102190 I_{n-4}+60385 I_{n-5}-19919 I_{n-6}+2824 I_{n-7}\right)
$$

and it holds:

$$
\theta_{n}-\left(y_{n}+\frac{1}{10} h^{2} y_{n}^{\prime \prime}\right)=O\left(h^{7}\right)
$$

In the next section, (7)-(9), (11) and (15) will be used as the artificial end conditions for integro interpolation; see (18) and (19).

\section{Integro Quintic Spline Interpolation with Five Artificial End Conditions}

In this section, we will use the given integral values (2) and the artificial end conditions in Section 2 to construct an integro quintic spline. Five additional independent conditions are needed. To use the results of (10) and (12) sufficiently, we will directly use the hybrid result of (15).

We look for the quintic spline $s$, which satisfies the following conditions:

$$
\begin{gathered}
\int_{x_{j}}^{x_{j+1}} s(x) d x=I_{j}, \quad j=0,1, \ldots, n-1, \\
s(a)=\widehat{y}_{0}, s^{\prime}(a)=\widehat{y}_{0}^{\prime}, s^{\prime \prime}(a)=\widehat{y}_{0}^{\prime \prime},
\end{gathered}
$$

and:

$$
s(b)+\frac{1}{10} h^{2} s^{\prime \prime}(b)=\theta_{n}, s^{\prime}(b)=\widehat{y}_{n}^{\prime} .
$$

It belongs to the spline space of $C^{4}$ quintic piecewise polynomial functions on the uniform partition $\Delta(1)$, so $s$ can be expressed as a linear combination of the quintic B-splines associated with the extended partition of $\Delta(1)$ with knots $a+i h,-5 \leq i \leq n+5$, i.e.,:

$$
s=\sum_{i=-2}^{n+2} c_{i} B_{i}
$$

where (see, e.g., $[6,14,20,21])$ :

$$
B_{i}(x)=\frac{1}{120 h^{5}} \begin{cases}\left(x-x_{i-3}\right)^{5}, & \text { if } x \in\left[x_{i-3}, x_{i-2}\right), \\ \left(x-x_{i-3}\right)^{5}-6\left(x-x_{i-2}\right)^{5}, & \text { if } x \in\left[x_{i-2}, x_{i-1}\right), \\ \left(x-x_{i-3}\right)^{5}-6\left(x-x_{i-2}\right)^{5}+15\left(x-x_{i-1}\right)^{5}, & \text { if } x \in\left[x_{i-1}, x_{i}\right), \\ \left(x_{i+3}-x\right)^{5}-6\left(x_{i+2}-x\right)^{5}+15\left(x_{i+1}-x\right)^{5}, & \text { if } x \in\left[x_{i}, x_{i+1}\right), \\ \left(x_{i+3}-x\right)^{5}-6\left(x_{i+2}-x\right)^{5}, & \text { if } x \in\left[x_{i+1}, x_{i+2}\right), \\ \left(x_{i+3}-x\right)^{5}, & \text { if } x \in\left[x_{i+2}, x_{i+3}\right), \\ 0, & \text { otherwise. }\end{cases}
$$


For the sake of completeness, we give in Table 1 the values of $B_{i}$ at the knots in $\left(x_{i-3}, x_{i+3}\right)$. Furthermore, we have the following integro properties:

$$
\begin{aligned}
\int_{x_{i-3}}^{x_{i-2}} B_{i}(x) d x & =\int_{x_{i+2}}^{x_{i+3}} B_{i}(x) d x=\frac{1}{720} h, \\
\int_{x_{i-2}}^{x_{i-1}} B_{i}(x) d x & =\int_{x_{i+1}}^{x_{i+2}} B_{i}(x) d x=\frac{57}{720} h, \\
\int_{x_{i-1}}^{x_{i}} B_{i}(x) d x & =\int_{x_{i}}^{x_{i+1}} B_{i}(x) d x=\frac{302}{720} h, \\
\int_{x_{j}}^{x_{j+1}} B_{i}(x) d x & =0, j \geq i+3 \text { or } j \leq i-4 .
\end{aligned}
$$

Table 1. The values of $B_{i}^{(k)}, k=0,1,2,3,4$, at the knots lying in the interior of the support of $B_{i}$.

\begin{tabular}{cccccc}
\hline$x$ & $x_{i-2}$ & $x_{i-1}$ & $x_{i}$ & $x_{i+1}$ & $x_{i+2}$ \\
\hline$B_{i}$ & $\frac{1}{120}$ & $\frac{26}{120}$ & $\frac{66}{120}$ & $\frac{26}{120}$ & $\frac{1}{120}$ \\
$B_{i}^{\prime}$ & $\frac{1}{24 h}$ & $\frac{10}{24 h}$ & 0 & $-\frac{10}{24 h}$ & $-\frac{1}{24 h}$ \\
$B_{i}^{\prime \prime}$ & $\frac{1}{6 h^{2}}$ & $\frac{2}{6 h^{2}}$ & $-\frac{6}{6 h^{2}}$ & $\frac{2}{6 h^{2}}$ & $\frac{1}{6 h^{2}}$ \\
$B_{i}^{(3)}$ & $\frac{1}{2 h^{3}}$ & $-\frac{2}{2 h^{3}}$ & 0 & $\frac{2}{2 h^{3}}$ & $-\frac{1}{2 h^{3}}$ \\
$B_{i}^{(4)}$ & $\frac{1}{h^{4}}$ & $-\frac{4}{h^{4}}$ & $\frac{6}{h^{4}}$ & $-\frac{4}{h^{4}}$ & $\frac{1}{h^{4}}$ \\
\hline
\end{tabular}

From (17) and (23), we have:

$$
\int_{x_{j}}^{x_{j+1}} s(x) d x=\sum_{i=j-2}^{j+3} c_{i} \int_{x_{j}}^{x_{j+1}} B_{i}(x) d x=I_{j} .
$$

Hence, for $j=0,1, \ldots, n-1$, by using (20)-(22), we get:

$$
\frac{h}{720}\left(c_{j-2}+57 c_{j-1}+302 c_{j}+302 c_{j+1}+57 c_{j+2}+c_{j+3}\right)=I_{j} .
$$

Since $s(a)=\widehat{y}_{0}$, it holds:

$$
c_{-2}+26 c_{-1}+66 c_{0}+26 c_{1}+c_{2}=120 \widehat{y}_{0} .
$$

The condition $s^{\prime}(a)=\widehat{y}_{0}^{\prime}$ provides the equality:

$$
-c_{-2}-10 c_{-1}+10 c_{1}+c_{2}=24 h \widehat{y}_{0}^{\prime} .
$$

Similarly, from $s^{\prime \prime}(a)=\widehat{y}_{0}^{\prime \prime}$, it follows that:

$$
c_{-2}+2 c_{-1}-6 c_{0}+2 c_{1}+c_{2}=6 h^{2} \widehat{y}_{0}^{\prime \prime} .
$$

Taking into account that $s(b)+\frac{1}{10} h^{2} s^{\prime \prime}(b)=\theta_{n}$, we get:

$$
\begin{aligned}
& \frac{1}{120}\left(c_{n-2}+26 c_{n-1}+66 c_{n}+26 c_{n+1}+c_{n+2}\right) \\
+ & \frac{h^{2}}{10} \cdot \frac{1}{6 h^{2}}\left(c_{n-2}+2 c_{n-1}-6 c_{n}+2 c_{n+1}+c_{n+2}\right)=\theta_{n},
\end{aligned}
$$


that is:

$$
\frac{1}{40}\left(c_{n-2}+10 c_{n-1}+18 c_{n}+10 c_{n+1}+c_{n+2}\right)=\theta_{n} .
$$

Finally, from $s^{\prime}(b)=\widehat{y}_{n}^{\prime}$, it follows that:

$$
-c_{n-2}-10 c_{n-1}+10 c_{n+1}+c_{n+2}=24 h \widehat{y}_{n}^{\prime} .
$$

Therefore, we get the linear system:

$$
A C=Y,
$$

where

$$
A=\left(\begin{array}{cccccccccc}
1 & 26 & 66 & 26 & 1 & & & & & \\
-1 & -10 & 0 & 10 & 1 & & & & & \\
1 & 2 & -6 & 2 & 1 & & & & & \\
1 & 57 & 302 & 302 & 57 & 1 & & & & \\
& 1 & 57 & 302 & 302 & 57 & 1 & & & \\
& & \ddots & \ddots & \ddots & \ddots & \ddots & \ddots & & \\
& & & 1 & 57 & 302 & 302 & 57 & 1 & \\
& & & & 1 & 57 & 302 & 302 & 57 & 1 \\
& & & & & 1 & 10 & 18 & 10 & 1 \\
& & & & & -1 & -10 & 0 & 10 & 1
\end{array}\right)_{(n+5) \times(n+5)}
$$

and:

$$
\begin{gathered}
C=\left(c_{-2}, c_{-1}, c_{0}, c_{1}, \cdots, c_{n+1}, c_{n+2}\right)^{T}, \\
Y=\left(120 \widehat{y}_{0}, 24 h \widehat{y}_{0}^{\prime}, 6 h^{2} \widehat{y}_{0}^{\prime \prime}, \frac{720}{h} I_{0}, \cdots, \frac{720}{h} I_{n-1}, 40 \theta_{n}, 24 h \widehat{y}_{n}^{\prime}\right)^{T} .
\end{gathered}
$$

Theorem 1. The coefficient matrix A (27) is invertible.

Proof. We will prove that the determinant of matrix $A$ is nonzero. We will perform some proper elementary transformations to $A$ in order to verify $|A| \neq 0$. Let $C(i)$ denote the $i$-th column and $R(i)$ denote the $i$-th row of a matrix obtained by an elementary row or column transformation.

We first perform $n+4$ elementary column transformations to $A$.

Step 1: For $i=n+4, n+3, \ldots, 1, C(i):=C(i)-C(i+1)$.

Then, we get:

$$
A_{1}=\left(\begin{array}{cccccccccc}
16 & -15 & 41 & 25 & 1 & & & & & \\
0 & -1 & -9 & 9 & 1 & & & & & \\
-8 & 9 & -7 & 1 & 1 & & & & & \\
0 & 1 & 56 & 246 & 56 & 1 & & & & \\
& 0 & 1 & 56 & 246 & 56 & 1 & & & \\
& & & \ddots & \ddots & \ddots & \ddots & \ddots & & \\
& & & & 1 & 56 & 246 & 56 & 1 & \\
& & & & & 1 & 56 & 246 & 56 & 1 \\
& & & & & 0 & 1 & 9 & 9 & 1 \\
& & & & & 0 & -1 & -9 & 9 & 1
\end{array}\right)_{(n+5) \times(n+5)} .
$$

We continue to perform the following elementary row transformations to $A_{1}$.

Step 2: $R(3):=\frac{2}{3} R(3)+\frac{1}{3} R(1)$; 
Step 3: $R(3):=R(3)+R(2)$, and $R(n+4):=R(n+4)-R(n+5)$;

Step 4: $R(4):=R(4)+R(2)$, and $R(n+3):=R(n+3)-R(n+5)$;

Step 5: $R(5):=R(5)-\frac{1}{47} R(4)$, and $R(n+2):=R(n+2)-\frac{1}{47} R(n+3)$;

Step 6: $R(3) \rightleftharpoons R(4)$, and $R(n+4) \rightleftharpoons R(n+3)$.

Thus, we get:

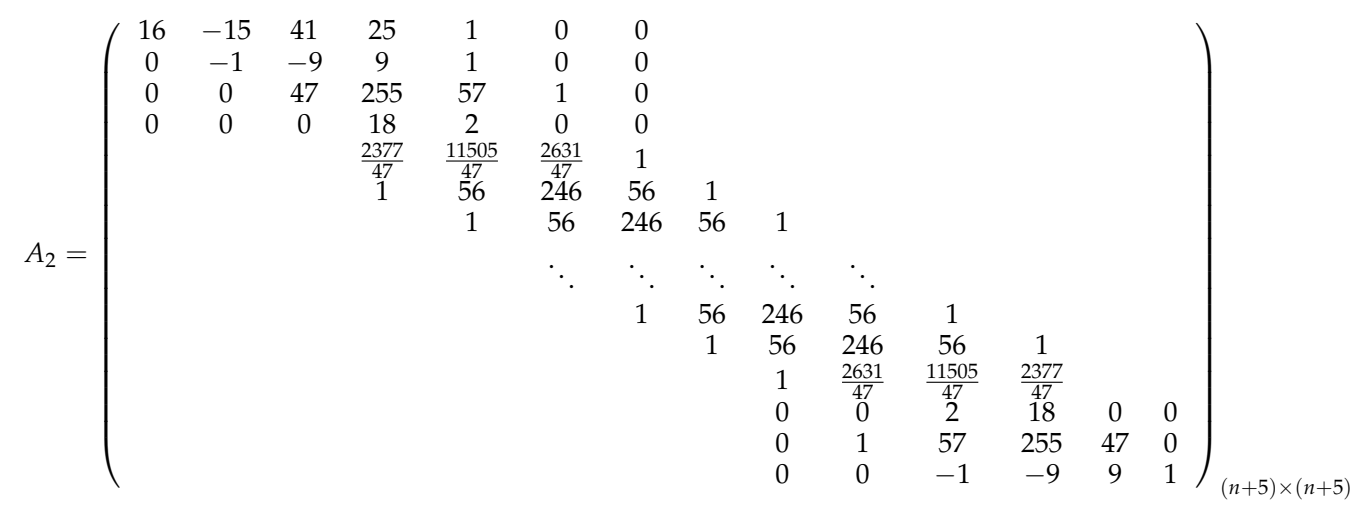

By the basic knowledge of linear algebra, we have:

$$
\left|A_{2}\right|=16 \times(-1) \times 47 \times\left|C_{n n}\right| \times 47 \times 1,
$$

where $C_{n n}$ is the central block matrix of $A_{2}$. $C_{n n}$ is strictly diagonally dominant, and so, $\left|C_{n n}\right| \neq 0$. It implies that $\left|A_{2}\right| \neq 0$ and, hence, $|A| \neq 0$. In other words, $A$ (27) is invertible, and the theorem is proven.

Theorem 1 guarantees the existence and uniqueness of the integro quintic spline $s=\sum_{i=-2}^{n+2} c_{i} B_{i}(x)$ determined by (17)-(19). It can be constructed as follows:

(I): Compute $\widehat{y}_{0}, \widehat{y}_{0}^{\prime}, \widehat{y}_{0}^{\prime \prime}, \widehat{y}_{n}^{\prime}$ and $\theta_{n}$ by using (7)-(9), (11) and (15), respectively;

(II): Solve the system (26) to get $c_{j}, j=-2,-1,0, \ldots, n+2$.

Evidently, the new method is free of exact end conditions and is easy to implement. Furthermore, the obtained quintic spline $s$ satisfies the conditions given in (2).

\section{Approximation Properties}

In this section, we study the approximation properties of the integro quintic spline $s$ obtained in Section 3.

For $k=0,1, \ldots, 5$, we use $y_{j}^{(k)}$ to denote $y^{(k)}\left(x_{j}\right), j=0,1, \ldots, n$. For $k=0,1,2,3,4$, we use $s_{j}, m_{j}$, $M_{j}, T_{j}$ and $F_{j}$ to denote $s^{(k)}\left(x_{j}\right), j=0,1, \ldots, n$. In addition, we define:

$$
W_{j}:=\frac{F_{j+1}-F_{j-1}}{2 h}
$$

in order to approximate $y^{(5)}\left(x_{j}\right), j=1,2, \ldots, n-1$. For $j=0,1, \ldots, n$,

$$
\begin{aligned}
s_{j} & =\frac{1}{120}\left(c_{j-2}+26 c_{j-1}+66 c_{j}+26 c_{j+1}+c_{j+2}\right), \\
m_{j} & =\frac{1}{24 h}\left(-c_{j-2}-10 c_{j-1}+10 c_{j+1}+c_{j+2}\right), \\
M_{j} & =\frac{1}{6 h^{2}}\left(c_{j-2}+2 c_{j-1}-6 c_{j}+2 c_{j+1}+c_{j+2}\right), \\
T_{j} & =\frac{1}{2 h^{3}}\left(-c_{j-2}+2 c_{j-1}-2 c_{j+1}+c_{j+2}\right), \\
F_{j} & =\frac{1}{h^{4}}\left(c_{j-2}-4 c_{j-1}+6 c_{j}-4 c_{j+1}+c_{j+2}\right) .
\end{aligned}
$$


Moreover,

$$
W_{j}=\frac{1}{2 h^{5}}\left(-c_{j-3}+4 c_{j-2}-5 c_{j-1}+5 c_{j+1}-4 c_{j+2}+c_{j+3}\right), j=1,2, \ldots, n-1 .
$$

By using (24), (25) and the above results, we can get some important relations between $s_{j}, m_{j}, M_{j}$, $T_{j}, F_{j}, W_{j}$ and $I_{j}$ of the integro quintic spline. We list the relations as follows.

(Set I)

$$
347 m_{0}+1044 m_{1}+225 m_{2}+4 m_{3}=\frac{1}{h^{2}}\left(120 I_{2}+1110 I_{1}-690 I_{0}\right)-\frac{540}{h} s_{0}-54 h M_{0} ;
$$

for $j=2,3, \ldots, n-2$,

$$
\begin{gathered}
m_{j-2}+56 m_{j-1}+246 m_{j}+56 m_{j+1}+m_{j+2}=\frac{30}{h^{2}}\left(-I_{j-2}-9 I_{j-1}+9 I_{j}+I_{j+1}\right) ; \\
4 m_{n-3}+225 m_{n-2}+1044 m_{n-1}+347 m_{n}=\frac{1}{h^{2}}\left(690 I_{n-1}-1110 I_{n-2}-120 I_{n-3}\right)+\frac{540}{h} \theta_{n} .
\end{gathered}
$$

(Set II)

For $j=0,1, \ldots, n-3$,

$$
T_{j}=\frac{2}{3 h^{2}}\left(28 m_{j}+245 m_{j+1}+56 m_{j+2}+m_{j+3}\right)+\frac{20}{h^{4}}\left(10 I_{j}-9 I_{j+1}-I_{j+2}\right) ;
$$

for $j=3,4, \ldots, n$,

$$
T_{j}=\frac{2}{3 h^{2}}\left(m_{j-3}+56 m_{j-2}+245 m_{j-1}+28 m_{j}\right)-\frac{20}{h^{4}}\left(10 I_{j-1}-9 I_{j-2}-I_{j-3}\right) .
$$

(Set III)

$$
\begin{gathered}
s_{1}=-s_{0}+\frac{1}{18 h}\left(47 I_{0}-10 I_{1}-I_{2}\right)+\frac{h}{540}\left(-61 m_{0}+363 m_{1}+57 m_{2}+m_{3}\right) ; \\
s_{2}=s_{0}+\frac{1}{3 h}\left(-8 I_{0}+8 I_{1}\right)+\frac{h}{9}\left(m_{0}-8 m_{1}+m_{2}\right)
\end{gathered}
$$

for $j=3,4, \ldots, n$,

$$
\begin{aligned}
s_{j}= & -s_{j-3}+\frac{1}{18 h}\left(47 I_{j-3}-58 I_{j-2}+47 I_{j-1}\right) \\
& +\frac{h}{540}\left(-61 m_{j-3}+423 m_{j-2}-423 m_{j-1}+61 m_{j}\right)
\end{aligned}
$$

(Set IV)

$$
M_{0}=\widehat{y}_{0}^{\prime \prime}=\frac{10}{h^{3}} I_{0}-\frac{10}{h^{2}} s_{0}-\frac{13}{3 h} m_{0}-\frac{2}{3 h} m_{1}-\frac{h}{9} T_{0}+\frac{h}{36} T_{1} ;
$$

for $j=1,2, \ldots, n$,

$$
M_{j}=-\frac{10}{h^{3}} I_{j-1}+\frac{10}{h^{2}} s_{j-1}+\frac{7}{3 h} m_{j-1}+\frac{8}{3 h} m_{j}-\frac{h}{18} T_{j-1}+\frac{5 h}{36} T_{j} .
$$

(Set V)

$$
F_{0}=-\frac{120}{h^{5}} I_{0}+\frac{120}{h^{4}} s_{0}+\frac{40}{h^{3}} m_{0}+\frac{20}{h^{3}} m_{1}-\frac{11}{3 h} T_{0}-\frac{4}{3 h} T_{1}
$$

for $j=1,2, \ldots, n$,

$$
F_{j}=\frac{120}{h^{5}} I_{j-1}-\frac{120}{h^{4}} s_{j-1}-\frac{40}{h^{3}} m_{j-1}-\frac{20}{h^{3}} m_{j}+\frac{5}{3 h} T_{j-1}+\frac{10}{3 h} T_{j} .
$$

(Set VI)

$$
W_{1}=\frac{60}{h^{6}}\left(I_{0}+I_{1}\right)-\frac{60}{h^{5}}\left(s_{0}+s_{1}\right)-\frac{10}{h^{4}}\left(2 m_{0}+3 m_{1}+m_{2}\right)+\frac{1}{6 h^{2}}\left(11 T_{0}+9 T_{1}+10 T_{2}\right) ;
$$


for $j=2,3, \ldots, n-1$,

$$
\begin{aligned}
W_{j}= & \frac{60}{h^{6}}\left(I_{j}-I_{j-2}\right)-\frac{60}{h^{5}}\left(s_{j}-s_{j-2}\right)-\frac{20}{h^{4}}\left(m_{j}-m_{j-2}\right) \\
& -\frac{10}{h^{4}}\left(m_{j+1}-m_{j-1}\right)+\frac{5}{6 h^{2}}\left(-T_{j-2}-2 T_{j-1}+T_{j}+2 T_{j+1}\right) .
\end{aligned}
$$

Theorem 2. Let s be the integro quintic spline determined by (17)-(19) with the artificial end conditions given in Section 2. For $j=0,1, \ldots, n$, we have:

$$
\begin{gathered}
s_{j}=y_{j}+O\left(h^{6}\right), \\
m_{j}=y_{j}^{\prime}+O\left(h^{6}\right), \\
M_{j}=y_{j}^{\prime \prime}+O\left(h^{4}\right), \\
T_{j}=y_{j}^{\prime \prime \prime}+O\left(h^{4}\right), \\
F_{j}=y_{j}^{(4)}+O\left(h^{2}\right) .
\end{gathered}
$$

For $j=1,2, \ldots, n-1$, we have:

$$
W_{j}=y_{j}^{(5)}+O\left(h^{2}\right)
$$

Proof. We first prove (43). We define $e_{j}^{\prime}:=m_{j}^{\prime}-y_{j}^{\prime}, j=0,1, \ldots, n$. From (28) and (13), we get:

$$
\begin{aligned}
& 347 e_{0}^{\prime}+1044 e_{1}^{\prime}+225 e_{2}^{\prime}+4 e_{3}^{\prime} \\
= & \left(347 m_{0}+1044 m_{1}+225 m_{2}+4 m_{3}\right)-\left(347 y_{0}^{\prime}+1044 y_{1}^{\prime}+225 y_{2}^{\prime}+4 y_{3}^{\prime}\right) \\
= & \frac{1}{h^{2}}\left(120 I_{2}+1110 I_{1}-690 I_{0}\right)-\frac{540}{h} \widehat{y}_{0}-54 h \widehat{y}_{0}^{\prime \prime}-\left(347 y_{0}^{\prime}+1044 y_{1}^{\prime}+225 y_{2}^{\prime}+4 y_{3}^{\prime}\right) \\
= & \frac{1}{h^{2}}\left\{-1800 I_{0}+990\left(I_{0}+I_{1}\right)+120\left(I_{0}+I_{1}+I_{2}\right)\right\} \\
& -\frac{540}{h}\left(y_{0}+O\left(h^{7}\right)\right)-54 h\left(y_{0}^{\prime \prime}+O\left(h^{5}\right)\right) \\
& -\left(347 y_{0}^{\prime}+1044 y_{1}^{\prime}+225 y_{2}^{\prime}+4 y_{3}^{\prime}\right)
\end{aligned}
$$

(continue to expand it at $x_{0}$ by using (4) and the Taylor formula)

$=O\left(h^{6}\right)$.

Similarly, from (30) and (14), it follows that:

$$
4 e_{n-3}^{\prime}+225 e_{n-2}^{\prime}+1044 e_{n-1}^{\prime}+347 e_{n}^{\prime}=O\left(h^{6}\right) .
$$

Besides, for $j=2,3, \ldots, n-2$, from (29), it follows that:

$$
\begin{aligned}
& e_{j-2}^{\prime}+56 e_{j-1}^{\prime}+246 e_{j}^{\prime}+56 e_{j+1}^{\prime}+e_{j+2}^{\prime} \\
= & \left(m_{j-2}+56 m_{j-1}+246 m_{j}+56 m_{j+1}+m_{j+2}\right)-\left(y_{j-2}^{\prime}+56 y_{j-1}^{\prime}+246 y_{j}^{\prime}+56 y_{j+1}^{\prime}+y_{j+2}^{\prime}\right) \\
= & \frac{30}{h^{2}}\left(-I_{j-2}-9 I_{j-1}+9 I_{j}+I_{j+1}\right)-\left(y_{j-2}^{\prime}+56 y_{j-1}^{\prime}+246 y_{j}^{\prime}+56 y_{j+1}^{\prime}+y_{j+2}^{\prime}\right) \\
= & \frac{30}{h^{2}}\left\{8 I_{j-2}-18\left(I_{j-2}+I_{j-1}\right)+8\left(I_{j-2}+I_{j-1}+I_{j}\right)+\left(I_{j-2}+I_{j-1}+I_{j}+I_{j+1}\right)\right\} \\
& -\left(y_{j-2}^{\prime}+56 y_{j-1}^{\prime}+246 y_{j}^{\prime}+56 y_{j+1}^{\prime}+y_{j+2}^{\prime}\right)
\end{aligned}
$$

(continue to expand it at $x_{j-2}$ by using a similar formula of (4) and the Taylor formula) $=O\left(h^{6}\right)$. 
Take into account:

$$
\begin{gathered}
e_{0}^{\prime}=m_{0}-y_{0}^{\prime}=\widehat{y}_{0}^{\prime}-y_{0}^{\prime}=O\left(h^{6}\right), \\
e_{n}^{\prime}=m_{n}-y_{n}^{\prime}=\widehat{y}_{n}^{\prime}-y_{n}^{\prime}=O\left(h^{6}\right),
\end{gathered}
$$

we get:

$$
\left(\begin{array}{ccccccc}
1 & & & & & & \\
347 & 1044 & 225 & 4 & & & \\
1 & 56 & 246 & 56 & 1 & & \\
& \ddots & \ddots & \ddots & \ddots & \ddots & \\
& & 1 & 56 & 246 & 56 & 1 \\
& & & 4 & 225 & 1044 & 347 \\
& & & & & & 1
\end{array}\right)\left(\begin{array}{c}
e_{0}^{\prime} \\
e_{1} \\
e_{2} \\
\vdots \\
e_{n-2}^{\prime} \\
e_{n-1}^{\prime} \\
e_{n}^{\prime}
\end{array}\right)=\left(\begin{array}{c}
O\left(h^{6}\right) \\
O\left(h^{6}\right) \\
O\left(h^{6}\right) \\
\vdots \\
O\left(h^{6}\right) \\
O\left(h^{6}\right) \\
O\left(h^{6}\right)
\end{array}\right)
$$

The coefficient matrix is strictly diagonally dominant. The infinity norm of its inverse is bounded. Hence, (43) is proven.

By using (31) and (43), we get:

$$
\begin{aligned}
T_{j}-y_{j}^{\prime \prime \prime}= & \frac{2}{3 h^{2}}\left(28\left(y_{j}^{\prime}+O\left(h^{6}\right)\right)+245\left(y_{j+1}^{\prime}+O\left(h^{6}\right)\right)+56\left(y_{j+2}^{\prime}+O\left(h^{6}\right)\right)\right. \\
& \left.+\left(y_{j+3}^{\prime}+O\left(h^{6}\right)\right)\right)+\frac{20}{h^{4}}\left(10 I_{j}-9 I_{j+1}-I_{j+2}\right)-y_{j}^{\prime \prime \prime} \\
= & O\left(h^{4}\right), j=0,1, \ldots, n-3 .
\end{aligned}
$$

It shows that (45) holds for $j=0,1, \ldots, n-3$. Similarly, by using (32) and (43), we get that (45) holds for $j=n-2, n-1, n$.

From (13), it follows that $s_{0}=\widehat{y}_{0}=y_{0}+O\left(h^{7}\right)$. By using (33)-(35), (43) and (45), we get $s_{j}-y_{j}=O\left(h^{6}\right), j=1,2, \ldots, n$. Therefore, $(42)$ is proven.

From (13), it follows that $M_{0}=\widehat{y}_{0}^{\prime \prime}=y_{0}^{\prime \prime}+O\left(h^{5}\right)$. Moreover, by using (37), (42), (43) and (45), we have:

$$
\begin{aligned}
M_{j}-y_{j}^{\prime \prime}= & -\frac{10}{h^{3}} I_{j-1}+\frac{10}{h^{2}}\left(y_{j-1}+O\left(h^{6}\right)\right)+\frac{7}{3 h}\left(y_{j-1}^{\prime}+O\left(h^{6}\right)\right) \\
& +\frac{8}{3 h}\left(y_{j}^{\prime}+O\left(h^{6}\right)\right)-\frac{h}{18}\left(y_{j-1}^{\prime \prime \prime}+O\left(h^{4}\right)\right)+\frac{5 h}{36}\left(y_{j}^{\prime \prime \prime}+O\left(h^{4}\right)\right)-y_{j}^{\prime \prime} \\
= & O\left(h^{4}\right), j=1,2, \ldots, n .
\end{aligned}
$$

Therefore, (44) is proven. In addition, (46) and (47) can be proven similarly by using (38)-(41) and (42), (43) and (45).

Theorem 2 shows that the new integro quintic spline has super convergence in locally approximating $y_{j}^{(k)}, k=1,3,5$, and full convergence in locally approximating $y_{j}^{(k)}, k=0,2,4$.

Theorem 3. Let s be the integro quintic spline determined by (17)-(19) with the artificial end conditions given in Section 2; we have:

$$
\left\|s^{(k)}(x)-y^{(k)}(x)\right\|_{\infty}=O\left(h^{6-k}\right), \quad k=0,1,2,3,4,5,
$$

where $\|\cdot\|_{\infty}:=\max _{a \leq x \leq b}|\cdot|$, and $s^{(5)}(x)$ is defined as follows:

$$
\begin{aligned}
s^{(5)}(x) & =\frac{F_{j+1}-F_{j}}{h}, \quad x_{j}<x<x_{j+1}, j=0,1,2, \ldots, n-1, \\
s^{(5)}\left(x_{0}\right) & =\frac{F_{1}-F_{0}}{h}, \\
s^{(5)}\left(x_{n}\right) & =\frac{F_{n}-F_{n-1}}{h}, \\
s^{(5)}\left(x_{j}\right) & =W_{j}, \quad j=1,2, \ldots, n-1 .
\end{aligned}
$$


Proof. By using (46), for $x \in\left(x_{j}, x_{j+1}\right), j=0,1,2, \ldots, n-1$,

$$
\begin{aligned}
s^{(5)}(x)-y^{(5)}(x) & =\frac{F_{j+1}-F_{j}}{h}-y^{(5)}(x) \\
& =\frac{y_{j+1}^{(4)}-y_{j}^{(4)}}{h}-y^{(5)}(x)+O(h) \\
& =y^{(5)}\left(\xi_{j}\right)-y^{(5)}(x)+O(h) \\
& =y^{(6)}\left(\eta_{j}\right)\left(\xi_{j}-x\right)+O(h) \\
& =O(h),
\end{aligned}
$$

where $\eta_{j}, \xi_{j} \in\left(x_{j}, x_{j+1}\right)$. Moreover, we have $s^{(5)}\left(x_{0}\right)-y_{0}^{(5)}=O(h), s^{(5)}\left(x_{n}\right)-y_{n}^{(5)}=O(h)$ and $s^{(5)}\left(x_{j}\right)-y_{j}^{(5)}=O\left(h^{2}\right), j=1,2, \ldots, n-1$. Hence,

$$
\left\|s^{(5)}(x)-y^{(5)}(x)\right\|_{\infty}=O(h) .
$$

Next, for $x \in\left[x_{j}, x_{j+1}\right], j=0,1,2, \ldots, n-1$,

$$
\begin{aligned}
s^{(4)}(x)-y^{(4)}(x) & =\left(F_{j}+\frac{F_{j+1}-F_{j}}{h}\left(x-x_{j}\right)\right)-\left(y_{j}^{(4)}+y_{j}^{(5)}\left(x-x_{j}\right)+O\left(h^{2}\right)\right) \\
& =O\left(h^{2}\right)+\left(\frac{F_{j+1}-F_{j}}{h}-y_{j}^{(5)}\right)\left(x-x_{j}\right) \\
& =O\left(h^{2}\right) .
\end{aligned}
$$

Hence, we get

$$
\left\|s^{(4)}(x)-y^{(4)}(x)\right\|_{\infty}=O\left(h^{2}\right) .
$$

The others also can be proven similarly. We omit the proof.

Theorem 3 shows that the new integro quintic spline has full convergence in globally approximating $y^{(k)}(x), k=0,1, \ldots, 5$.

\section{Numerical Tests}

In this section, we test the approximation properties of the new integro quintic spline. Our tests are performed by MATLAB.

We take:

$$
y_{1}=e^{x}, \quad x \in[0,1]
$$

and:

$$
y_{2}= \begin{cases}\sin x, & \text { if } x \in[-0.5,0), \\ x-\frac{x^{3}}{3 !}+\frac{x^{5}}{5 !}-\frac{x^{7}}{7 !}, & \text { if } x \in[0,0.5],\end{cases}
$$

as two illustrative examples. Furthermore, $y_{1}$ will be used in the comparison of our method with some other methods.

The absolute errors at the knots are defined as follows:

$$
E_{k}\left(x_{i}, n\right):=\left|y^{(k)}\left(x_{i}\right)-s^{(k)}\left(x_{i}\right)\right|, \quad k=0,1,2,3,4, \quad i=0,1, \ldots, n,
$$

and:

$$
E_{5}\left(x_{i}, n\right):=\left|y^{(5)}\left(x_{i}\right)-\frac{s^{(4)}\left(x_{i+1}\right)-s^{(4)}\left(x_{i-1}\right)}{2 h}\right|, \quad i=1,2, \ldots, n-1
$$


The numerical convergence orders of the absolute errors at the knots are defined by:

$$
O_{k}\left(x_{i}, n_{1}, n_{2}\right):=\frac{\log \left(E_{k}\left(x_{i}, n_{1}\right) / E_{k}\left(x_{i}, n_{2}\right)\right)}{\log \left(n_{2} / n_{1}\right)}, \quad k=0,1, \ldots, 5 .
$$

Tables 2-7 show the absolute errors $E_{k}\left(x_{i}, n\right)$ of $y_{1}^{(k)}(x)$ at the chosen knots and the numerical convergence orders $O_{k}\left(x_{i}, n_{1}, n_{2}\right)$, where $n=10,20,40, k=0,1, \ldots, 5$. The results of $y_{2}^{(k)}(x)$ are given in Tables 8-13.

The numerical convergence orders in these tables accord with the theoretical expectation. By Theorem 2, $E_{0}\left(x_{i}, n\right)$ and $E_{1}\left(x_{i}, n\right)$ are of sixth order convergent (see Tables 2, 3, 8 and 9 for the numerical convergence orders), $E_{2}\left(x_{i}, n\right)$ and $E_{3}\left(x_{i}, n\right)$ are of fourth order convergent (see Tables 4,5 , 10 and 11 for the numerical convergence orders), $E_{4}\left(x_{i}, n\right)$ and $E_{5}\left(x_{i}, n\right)$ are of second order convergent (see Tables 6, 7, 12 and 13 for the numerical convergence orders).

Table 2. The absolute errors of the function values of $y_{1}$ at the knots and the numerical convergence orders.

\begin{tabular}{cccccc}
\hline$x_{\boldsymbol{i}}$ & $\boldsymbol{E}_{\mathbf{0}}\left(\boldsymbol{x}_{\boldsymbol{i}}, \mathbf{1 0}\right)$ & $\boldsymbol{E}_{\mathbf{0}}\left(\boldsymbol{x}_{\boldsymbol{i}}, \mathbf{2 0}\right)$ & $\boldsymbol{E}_{\mathbf{0}}\left(\boldsymbol{x}_{\boldsymbol{i}}, \mathbf{4 0}\right)$ & $\boldsymbol{O}_{\mathbf{0}}\left(\boldsymbol{x}_{\boldsymbol{i}}, \mathbf{1 0} \mathbf{2 0}\right)$ & $\boldsymbol{O}_{\mathbf{0}}\left(\boldsymbol{x}_{\boldsymbol{i}}, \mathbf{1 0}, \mathbf{4 0}\right)$ \\
\hline 0.0 & $1.711 \times 10^{-8}$ & $1.141 \times 10^{-10}$ & $7.632 \times 10^{-13}$ & 7.2 & 7.2 \\
0.1 & $2.512 \times 10^{-9}$ & $4.480 \times 10^{-12}$ & $2.887 \times 10^{-15}$ & 9.1 & 9.8 \\
0.2 & $7.533 \times 10^{-10}$ & $1.627 \times 10^{-12}$ & $7.105 \times 10^{-15}$ & 8.9 & 8.3 \\
0.3 & $4.974 \times 10^{-10}$ & $1.433 \times 10^{-12}$ & $1.998 \times 10^{-15}$ & 8.4 & 8.9 \\
0.4 & $3.287 \times 10^{-10}$ & $1.351 \times 10^{-12}$ & $1.799 \times 10^{-14}$ & 7.9 & 7.1 \\
0.5 & $4.105 \times 10^{-10}$ & $1.277 \times 10^{-12}$ & $5.107 \times 10^{-15}$ & 8.3 & 8.1 \\
0.6 & $2.701 \times 10^{-10}$ & $1.187 \times 10^{-12}$ & $5.773 \times 10^{-15}$ & 7.8 & 7.7 \\
0.7 & $2.914 \times 10^{-10}$ & $1.075 \times 10^{-12}$ & $1.954 \times 10^{-14}$ & 8.1 & 6.9 \\
0.8 & $3.233 \times 10^{-10}$ & $7.208 \times 10^{-13}$ & $1.643 \times 10^{-14}$ & 8.8 & 7.1 \\
0.9 & $2.535 \times 10^{-9}$ & $4.620 \times 10^{-12}$ & $3.108 \times 10^{-15}$ & 9.1 & 9.7 \\
1.0 & $2.403 \times 10^{-8}$ & $2.195 \times 10^{-10}$ & $1.720 \times 10^{-12}$ & 6.8 & 6.9 \\
\hline
\end{tabular}

Table 3. The absolute errors of the first order derivatives of $y_{1}$ at the knots and the numerical convergence orders.

\begin{tabular}{cccccc}
\hline $\boldsymbol{x}_{\boldsymbol{i}}$ & $\boldsymbol{E}_{\mathbf{1}}\left(\boldsymbol{x}_{\boldsymbol{i}}, \mathbf{1 0}\right)$ & $\boldsymbol{E}_{\mathbf{1}}\left(\boldsymbol{x}_{\boldsymbol{i}}, \mathbf{2 0}\right)$ & $\boldsymbol{E}_{\mathbf{1}}\left(\boldsymbol{x}_{\boldsymbol{i}}, \mathbf{4 0}\right)$ & $\boldsymbol{O}_{\mathbf{1}}\left(\boldsymbol{x}_{\boldsymbol{i}}, \mathbf{1 0} \mathbf{2 0}\right)$ & $\boldsymbol{O}_{\mathbf{1}}\left(\boldsymbol{x}_{\boldsymbol{i}}, \mathbf{1 0}, \mathbf{4 0}\right)$ \\
\hline 0.0 & $8.837 \times 10^{-7}$ & $1.181 \times 10^{-8}$ & $1.599 \times 10^{-10}$ & 6.2 & 6.2 \\
0.1 & $7.198 \times 10^{-8}$ & $1.782 \times 10^{-10}$ & $6.932 \times 10^{-13}$ & 8.7 & 8.4 \\
0.2 & $1.321 \times 10^{-8}$ & $5.626 \times 10^{-12}$ & $9.104 \times 10^{-15}$ & 11.2 & 10.1 \\
0.3 & $3.138 \times 10^{-9}$ & $3.064 \times 10^{-12}$ & $6.535 \times 10^{-13}$ & 10.0 & 6.1 \\
0.4 & $4.357 \times 10^{-10}$ & $3.753 \times 10^{-12}$ & $2.014 \times 10^{-13}$ & 6.9 & 5.5 \\
0.5 & $6.093 \times 10^{-10}$ & $4.163 \times 10^{-12}$ & $2.633 \times 10^{-13}$ & 7.2 & 5.5 \\
0.6 & $6.225 \times 10^{-10}$ & $4.451 \times 10^{-12}$ & $4.776 \times 10^{-13}$ & 7.1 & 5.1 \\
0.7 & $4.399 \times 10^{-9}$ & $4.471 \times 10^{-12}$ & $1.275 \times 10^{-13}$ & 9.9 & 7.5 \\
0.8 & $1.839 \times 10^{-8}$ & $1.034 \times 10^{-11}$ & $2.132 \times 10^{-13}$ & 10.8 & 8.1 \\
0.9 & $1.020 \times 10^{-7}$ & $3.315 \times 10^{-10}$ & $9.912 \times 10^{-13}$ & 8.3 & 8.3 \\
1.0 & $1.300 \times 10^{-6}$ & $2.363 \times 10^{-8}$ & $3.788 \times 10^{-10}$ & 5.8 & 5.8 \\
\hline
\end{tabular}


Table 4. The absolute errors of the second order derivatives of $y_{1}$ at the knots and the numerical convergence orders.

\begin{tabular}{cccccc}
\hline$x_{\boldsymbol{i}}$ & $\boldsymbol{E}_{\mathbf{2}}\left(\boldsymbol{x}_{\boldsymbol{i}}, \mathbf{1 0}\right)$ & $\boldsymbol{E}_{\mathbf{2}}\left(\boldsymbol{x}_{\boldsymbol{i}}, \mathbf{2 0}\right)$ & $\boldsymbol{E}_{\mathbf{2}}\left(\boldsymbol{x}_{\boldsymbol{i}}, \mathbf{4 0}\right)$ & $\boldsymbol{O}_{\mathbf{2}}\left(\boldsymbol{x}_{\boldsymbol{i}}, \mathbf{1 0}, \mathbf{2 0}\right)$ & $\boldsymbol{O}_{\mathbf{2}}\left(\boldsymbol{x}_{\boldsymbol{i}}, \mathbf{1 0}, \mathbf{4 0}\right)$ \\
\hline 0.0 & $2.647 \times 10^{-5}$ & $7.099 \times 10^{-7}$ & $1.949 \times 10^{-8}$ & 5.2 & 5.2 \\
0.1 & $4.869 \times 10^{-7}$ & $1.820 \times 10^{-9}$ & $3.831 \times 10^{-10}$ & 8.1 & 5.1 \\
0.2 & $2.978 \times 10^{-7}$ & $1.957 \times 10^{-9}$ & $4.236 \times 10^{-10}$ & 7.2 & 4.7 \\
0.3 & $5.713 \times 10^{-7}$ & $3.211 \times 10^{-9}$ & $5.213 \times 10^{-10}$ & 7.5 & 5.0 \\
0.4 & $1.569 \times 10^{-7}$ & $4.450 \times 10^{-9}$ & $3.785 \times 10^{-10}$ & 5.1 & 4.3 \\
0.5 & $5.861 \times 10^{-7}$ & $5.800 \times 10^{-9}$ & $6.002 \times 10^{-10}$ & 6.7 & 4.9 \\
0.6 & $9.784 \times 10^{-8}$ & $7.297 \times 10^{-9}$ & $7.447 \times 10^{-10}$ & 3.7 & 3.5 \\
0.7 & $6.007 \times 10^{-7}$ & $8.972 \times 10^{-9}$ & $5.578 \times 10^{-10}$ & 6.1 & 5.0 \\
0.8 & $1.011 \times 10^{-7}$ & $1.108 \times 10^{-8}$ & $7.138 \times 10^{-10}$ & 3.2 & 3.5 \\
0.9 & $7.946 \times 10^{-7}$ & $1.825 \times 10^{-8}$ & $8.472 \times 10^{-10}$ & 5.4 & 4.9 \\
1.0 & $4.041 \times 10^{-5}$ & $1.462 \times 10^{-6}$ & $4.772 \times 10^{-8}$ & 4.8 & 4.8 \\
\hline
\end{tabular}

Table 5. The absolute errors of the third order derivatives of $y_{1}$ at the knots and the numerical convergence orders.

\begin{tabular}{cccccc}
\hline$x_{\boldsymbol{i}}$ & $E_{\mathbf{3}}\left(\boldsymbol{x}_{\boldsymbol{i}}, \mathbf{1 0}\right)$ & $E_{\mathbf{3}}\left(\boldsymbol{x}_{\boldsymbol{i}}, \mathbf{2 0}\right)$ & $E_{\mathbf{3}}\left(\boldsymbol{x}_{\boldsymbol{i}}, \mathbf{4 0}\right)$ & $\boldsymbol{O}_{\mathbf{3}}\left(\boldsymbol{x}_{\boldsymbol{i}}, \mathbf{1 0}, \mathbf{2 0}\right)$ & $\boldsymbol{O}_{\mathbf{3}}\left(\boldsymbol{x}_{\boldsymbol{i}}, \mathbf{1 0}, \mathbf{4 0}\right)$ \\
\hline 0.0 & $5.275 \times 10^{-4}$ & $2.780 \times 10^{-5}$ & $1.471 \times 10^{-6}$ & 4.2 & 4.2 \\
0.1 & $7.466 \times 10^{-5}$ & $1.054 \times 10^{-6}$ & $5.170 \times 10^{-9}$ & 6.1 & 6.8 \\
0.2 & $1.955 \times 10^{-5}$ & $2.196 \times 10^{-8}$ & $2.435 \times 10^{-9}$ & 9.8 & 6.4 \\
0.3 & $5.064 \times 10^{-6}$ & $3.244 \times 10^{-8}$ & $2.362 \times 10^{-9}$ & 7.3 & 5.5 \\
0.4 & $4.352 \times 10^{-7}$ & $3.852 \times 10^{-8}$ & $2.592 \times 10^{-10}$ & 3.5 & 5.3 \\
0.5 & $1.209 \times 10^{-6}$ & $4.265 \times 10^{-8}$ & $5.388 \times 10^{-9}$ & 4.8 & 3.9 \\
0.6 & $6.714 \times 10^{-7}$ & $4.702 \times 10^{-8}$ & $1.748 \times 10^{-9}$ & 3.8 & 4.2 \\
0.7 & $7.116 \times 10^{-6}$ & $4.759 \times 10^{-8}$ & $1.095 \times 10^{-9}$ & 7.2 & 6.3 \\
0.8 & $2.705 \times 10^{-5}$ & $4.163 \times 10^{-8}$ & $4.917 \times 10^{-9}$ & 9.3 & 6.1 \\
0.9 & $1.027 \times 10^{-4}$ & $1.950 \times 10^{-6}$ & $9.570 \times 10^{-9}$ & 5.7 & 6.6 \\
1.0 & $8.400 \times 10^{-4}$ & $6.182 \times 10^{-5}$ & $4.229 \times 10^{-6}$ & 3.8 & 3.8 \\
\hline
\end{tabular}

Table 6. The absolute errors of the fourth order derivatives of $y_{1}$ at the knots and the numerical convergence orders.

\begin{tabular}{cccccc}
\hline$x_{\boldsymbol{i}}$ & $E_{\mathbf{4}}\left(\boldsymbol{x}_{\boldsymbol{i}}, \mathbf{1 0}\right)$ & $E_{\mathbf{4}}\left(\boldsymbol{x}_{\boldsymbol{i}}, \mathbf{2 0}\right)$ & $E_{\mathbf{4}}\left(\boldsymbol{x}_{\boldsymbol{i}}, \mathbf{4 0}\right)$ & $\boldsymbol{O}_{\mathbf{4}}\left(\boldsymbol{x}_{\boldsymbol{i}}, \mathbf{1 0}, \mathbf{2 0}\right)$ & $\boldsymbol{O}_{\mathbf{4}}\left(\boldsymbol{x}_{\boldsymbol{i}}, \mathbf{1 0}, \mathbf{4 0}\right)$ \\
\hline 0.0 & $6.139 \times 10^{-3}$ & $5.105 \times 10^{-4}$ & $2.006 \times 10^{-5}$ & 3.6 & 4.0 \\
0.1 & $1.166 \times 10^{-3}$ & $2.577 \times 10^{-4}$ & $5.397 \times 10^{-5}$ & 2.2 & 2.2 \\
0.2 & $1.219 \times 10^{-3}$ & $2.171 \times 10^{-4}$ & $5.919 \times 10^{-5}$ & 2.5 & 2.0 \\
0.3 & $1.414 \times 10^{-3}$ & $2.406 \times 10^{-4}$ & $6.602 \times 10^{-5}$ & 2.6 & 2.1 \\
0.4 & $8.417 \times 10^{-4}$ & $2.700 \times 10^{-4}$ & $7.006 \times 10^{-5}$ & 1.6 & 1.8 \\
0.5 & $1.806 \times 10^{-3}$ & $3.026 \times 10^{-4}$ & $8.012 \times 10^{-5}$ & 2.6 & 2.2 \\
0.6 & $1.046 \times 10^{-3}$ & $3.387 \times 10^{-4}$ & $8.963 \times 10^{-5}$ & 1.6 & 1.7 \\
0.7 & $2.303 \times 10^{-3}$ & $3.783 \times 10^{-4}$ & $9.521 \times 10^{-5}$ & 2.6 & 2.2 \\
0.8 & $5.430 \times 10^{-4}$ & $4.165 \times 10^{-4}$ & $1.067 \times 10^{-4}$ & 0.4 & 1.1 \\
0.9 & $5.952 \times 10^{-3}$ & $3.446 \times 10^{-4}$ & $1.174 \times 10^{-4}$ & 4.1 & 2.8 \\
1.0 & $1.311 \times 10^{-2}$ & $2.204 \times 10^{-3}$ & $3.719 \times 10^{-4}$ & 2.6 & 2.6 \\
\hline
\end{tabular}


Table 7. The absolute errors of the fifth order derivatives of $y_{1}$ at the knots and the numerical convergence orders.

\begin{tabular}{cccccc}
\hline$x_{\boldsymbol{i}}$ & $E_{\mathbf{5}}\left(\boldsymbol{x}_{\boldsymbol{i}}, \mathbf{1 0}\right)$ & $E_{\mathbf{5}}\left(\boldsymbol{x}_{\boldsymbol{i}}, \mathbf{2 0}\right)$ & $E_{\mathbf{5}}\left(\boldsymbol{x}_{\boldsymbol{i}}, \mathbf{4 0}\right)$ & $\boldsymbol{O}_{\mathbf{5}}\left(\boldsymbol{x}_{\boldsymbol{i}}, \mathbf{1 0}, \mathbf{2 0}\right)$ & $\boldsymbol{O}_{\mathbf{5}}\left(\boldsymbol{x}_{\boldsymbol{i}}, \mathbf{1 0}, \mathbf{4 0}\right)$ \\
\hline 0.1 & $3.494 \times 10^{-2}$ & $2.340 \times 10^{-3}$ & $3.900 \times 10^{-6}$ & 3.9 & 6.5 \\
0.2 & $1.086 \times 10^{-2}$ & $1.096 \times 10^{-4}$ & $6.875 \times 10^{-5}$ & 6.6 & 3.6 \\
0.3 & $4.136 \times 10^{-3}$ & $2.740 \times 10^{-4}$ & $4.483 \times 10^{-5}$ & 3.9 & 3.3 \\
0.4 & $5.293 \times 10^{-4}$ & $3.101 \times 10^{-4}$ & $6.563 \times 10^{-5}$ & 0.8 & 1.5 \\
0.5 & $1.727 \times 10^{-3}$ & $3.430 \times 10^{-4}$ & $1.049 \times 10^{-4}$ & 2.3 & 2.0 \\
0.6 & $5.522 \times 10^{-4}$ & $3.787 \times 10^{-4}$ & $6.581 \times 10^{-5}$ & 0.5 & 1.5 \\
0.7 & $5.874 \times 10^{-3}$ & $4.064 \times 10^{-4}$ & $8.943 \times 10^{-5}$ & 3.9 & 3.0 \\
0.8 & $1.453 \times 10^{-2}$ & $1.946 \times 10^{-4}$ & $1.241 \times 10^{-4}$ & 6.2 & 3.4 \\
0.9 & $5.871 \times 10^{-2}$ & $4.198 \times 10^{-3}$ & $1.931 \times 10^{-5}$ & 3.8 & 5.7 \\
\hline
\end{tabular}

Table 8. The absolute errors of the function values of $y_{2}$ at the knots and the numerical convergence orders.

\begin{tabular}{cccccc}
\hline $\boldsymbol{x}_{\boldsymbol{i}}$ & $\boldsymbol{E}_{\mathbf{0}}\left(\boldsymbol{x}_{\boldsymbol{i}}, \mathbf{1 0}\right)$ & $\boldsymbol{E}_{\mathbf{0}}\left(\boldsymbol{x}_{\boldsymbol{i}}, \mathbf{2 0}\right)$ & $\boldsymbol{E}_{\mathbf{0}}\left(\boldsymbol{x}_{\boldsymbol{i}}, \mathbf{4 0}\right)$ & $\boldsymbol{O}_{\mathbf{0}}\left(\boldsymbol{x}_{\boldsymbol{i}}, \mathbf{1 0}, \mathbf{2 0}\right)$ & $\boldsymbol{O}_{\mathbf{0}}\left(\boldsymbol{x}_{\boldsymbol{i}}, \mathbf{1 0}, \mathbf{4 0}\right)$ \\
\hline-0.5 & $1.224 \times 10^{-8}$ & $9.184 \times 10^{-11}$ & $7.080 \times 10^{-13}$ & 7.1 & 7.0 \\
-0.4 & $1.748 \times 10^{-9}$ & $4.632 \times 10^{-12}$ & $1.765 \times 10^{-14}$ & 8.6 & 8.2 \\
-0.3 & $5.906 \times 10^{-10}$ & $2.425 \times 10^{-12}$ & $1.460 \times 10^{-14}$ & 7.9 & 7.6 \\
-0.2 & $3.360 \times 10^{-10}$ & $2.273 \times 10^{-12}$ & $5.940 \times 10^{-15}$ & 7.2 & 7.8 \\
-0.1 & $2.934 \times 10^{-10}$ & $2.220 \times 10^{-12}$ & $1.117 \times 10^{-14}$ & 7.0 & 7.3 \\
0.0 & $2.761 \times 10^{-10}$ & $2.167 \times 10^{-12}$ & $6.559 \times 10^{-15}$ & 7.0 & 7.6 \\
0.1 & $2.586 \times 10^{-10}$ & $2.116 \times 10^{-12}$ & $6.231 \times 10^{-15}$ & 6.9 & 7.6 \\
0.2 & $2.150 \times 10^{-10}$ & $2.057 \times 10^{-12}$ & $4.469 \times 10^{-15}$ & 6.7 & 7.7 \\
0.3 & $4.428 \times 10^{-11}$ & $1.895 \times 10^{-12}$ & $4.385 \times 10^{-15}$ & 4.5 & 6.6 \\
0.4 & $1.225 \times 10^{-9}$ & $4.481 \times 10^{-13}$ & $2.498 \times 10^{-15}$ & 11.4 & 9.4 \\
0.5 & $1.194 \times 10^{-8}$ & $9.321 \times 10^{-11}$ & $7.356 \times 10^{-13}$ & 7.0 & 6.9 \\
\hline
\end{tabular}

Table 9. The absolute errors of the first order derivatives of $y_{2}$ at the knots and the numerical convergence orders.

\begin{tabular}{cccccc}
\hline$x_{\boldsymbol{i}}$ & $\boldsymbol{E}_{\mathbf{1}}\left(\boldsymbol{x}_{\boldsymbol{i}}, \mathbf{1 0}\right)$ & $\boldsymbol{E}_{\mathbf{1}}\left(\boldsymbol{x}_{\boldsymbol{i}}, \mathbf{2 0}\right)$ & $\boldsymbol{E}_{\mathbf{1}}\left(\boldsymbol{x}_{\boldsymbol{i}}, \mathbf{4 0}\right)$ & $\boldsymbol{O}_{\mathbf{1}}\left(\boldsymbol{x}_{\boldsymbol{i}}, \mathbf{1 0}, \mathbf{2 0}\right)$ & $\boldsymbol{O}_{\mathbf{1}}\left(\boldsymbol{x}_{\boldsymbol{i}}, \mathbf{1 0}, \mathbf{4 0}\right)$ \\
\hline-0.5 & $6.342 \times 10^{-7}$ & $9.518 \times 10^{-9}$ & $1.470 \times 10^{-10}$ & 6.0 & 6.0 \\
-0.4 & $5.069 \times 10^{-8}$ & $1.374 \times 10^{-10}$ & $7.450 \times 10^{-14}$ & 8.5 & 9.6 \\
-0.3 & $9.230 \times 10^{-9}$ & $4.217 \times 10^{-12}$ & $2.941 \times 10^{-13}$ & 11.0 & 7.4 \\
-0.2 & $2.200 \times 10^{-9}$ & $2.199 \times 10^{-12}$ & $7.871 \times 10^{-14}$ & 9.9 & 7.3 \\
-0.1 & $3.042 \times 10^{-10}$ & $2.467 \times 10^{-12}$ & $1.760 \times 10^{-13}$ & 6.9 & 5.3 \\
0.0 & $3.641 \times 10^{-10}$ & $2.618 \times 10^{-12}$ & $1.416 \times 10^{-13}$ & 7.1 & 5.6 \\
0.1 & $3.119 \times 10^{-10}$ & $2.557 \times 10^{-12}$ & $5.085 \times 10^{-14}$ & 6.9 & 6.2 \\
0.2 & $2.242 \times 10^{-9}$ & $2.245 \times 10^{-12}$ & $6.439 \times 10^{-15}$ & 9.9 & 9.2 \\
0.3 & $9.406 \times 10^{-9}$ & $4.439 \times 10^{-12}$ & $1.124 \times 10^{-13}$ & 11.0 & 8.1 \\
0.4 & $5.172 \times 10^{-8}$ & $1.459 \times 10^{-10}$ & $4.774 \times 10^{-14}$ & 8.4 & 10.0 \\
0.5 & $6.482 \times 10^{-7}$ & $1.013 \times 10^{-8}$ & $1.584 \times 10^{-10}$ & 6.0 & 5.9 \\
\hline
\end{tabular}


Table 10. The absolute errors of the second order derivatives of $y_{2}$ at the knots and the numerical convergence orders.

\begin{tabular}{cccccc}
\hline$x_{\boldsymbol{i}}$ & $\boldsymbol{E}_{\mathbf{2}}\left(\boldsymbol{x}_{\boldsymbol{i}}, \mathbf{1 0}\right)$ & $\boldsymbol{E}_{\mathbf{2}}\left(\boldsymbol{x}_{\boldsymbol{i}}, \mathbf{2 0}\right)$ & $\boldsymbol{E}_{\mathbf{2}}\left(\boldsymbol{x}_{\boldsymbol{i}}, \mathbf{4 0}\right)$ & $\boldsymbol{O}_{\mathbf{2}}\left(\boldsymbol{x}_{\boldsymbol{i}}, \mathbf{1 0}, \mathbf{2 0}\right)$ & $\boldsymbol{O}_{\mathbf{2}}\left(\boldsymbol{x}_{\boldsymbol{i}}, \mathbf{1 0}, \mathbf{4 0}\right)$ \\
\hline-0.5 & $1.909 \times 10^{-5}$ & $5.731 \times 10^{-7}$ & $1.765 \times 10^{-8}$ & 5.0 & 5.0 \\
-0.4 & $1.899 \times 10^{-7}$ & $1.429 \times 10^{-8}$ & $4.735 \times 10^{-10}$ & 3.7 & 4.3 \\
-0.3 & $3.931 \times 10^{-7}$ & $1.135 \times 10^{-8}$ & $3.656 \times 10^{-10}$ & 5.1 & 5.0 \\
-0.2 & $2.668 \times 10^{-7}$ & $1.039 \times 10^{-8}$ & $2.035 \times 10^{-10}$ & 4.6 & 5.1 \\
-0.1 & $2.939 \times 10^{-7}$ & $9.536 \times 10^{-9}$ & $1.882 \times 10^{-10}$ & 4.9 & 5.3 \\
0.0 & $2.761 \times 10^{-7}$ & $8.669 \times 10^{-9}$ & $1.213 \times 10^{-10}$ & 4.9 & 5.5 \\
0.1 & $2.583 \times 10^{-7}$ & $7.801 \times 10^{-9}$ & $5.797 \times 10^{-11}$ & 5.0 & 6.0 \\
0.2 & $2.853 \times 10^{-7}$ & $6.926 \times 10^{-9}$ & $2.573 \times 10^{-12}$ & 5.3 & 8.3 \\
0.3 & $1.572 \times 10^{-7}$ & $5.933 \times 10^{-9}$ & $3.983 \times 10^{-11}$ & 4.7 & 5.9 \\
0.4 & $3.659 \times 10^{-7}$ & $2.799 \times 10^{-9}$ & $1.204 \times 10^{-10}$ & 7.0 & 5.7 \\
0.5 & $2.010 \times 10^{-5}$ & $6.285 \times 10^{-7}$ & $1.954 \times 10^{-8}$ & 4.9 & 5.0 \\
\hline
\end{tabular}

Table 11. The absolute errors of the third order derivatives of $y_{2}$ at the knots and the numerical convergence orders.

\begin{tabular}{cccccc}
\hline$x_{\boldsymbol{i}}$ & $E_{\mathbf{3}}\left(\boldsymbol{x}_{\boldsymbol{i}}, \mathbf{1 0}\right)$ & $E_{\mathbf{3}}\left(\boldsymbol{x}_{\boldsymbol{i}}, \mathbf{2 0}\right)$ & $\boldsymbol{E}_{\mathbf{3}}\left(\boldsymbol{x}_{\boldsymbol{i}}, \mathbf{4 0}\right)$ & $\boldsymbol{O}_{\mathbf{3}}\left(\boldsymbol{x}_{\boldsymbol{i}}, \mathbf{1 0}, \mathbf{2 0}\right)$ & $\boldsymbol{O}_{\mathbf{3}}\left(\boldsymbol{x}_{\boldsymbol{i}}, \mathbf{1 0}, \mathbf{4 0}\right)$ \\
\hline-0.5 & $3.940 \times 10^{-4}$ & $2.394 \times 10^{-5}$ & $1.498 \times 10^{-6}$ & 4.0 & 4.0 \\
-0.4 & $5.180 \times 10^{-5}$ & $8.104 \times 10^{-7}$ & $5.789 \times 10^{-10}$ & 5.9 & 8.2 \\
-0.3 & $1.362 \times 10^{-5}$ & $1.640 \times 10^{-8}$ & $4.348 \times 10^{-9}$ & 9.6 & 5.8 \\
-0.2 & $3.555 \times 10^{-6}$ & $2.343 \times 10^{-8}$ & $2.673 \times 10^{-9}$ & 7.2 & 5.1 \\
-0.1 & $3.114 \times 10^{-7}$ & $2.567 \times 10^{-8}$ & $8.109 \times 10^{-11}$ & 3.6 & 5.9 \\
0.0 & $7.246 \times 10^{-7}$ & $2.607 \times 10^{-8}$ & $2.454 \times 10^{-9}$ & 4.7 & 4.1 \\
0.1 & $3.225 \times 10^{-7}$ & $2.594 \times 10^{-8}$ & $1.845 \times 10^{-9}$ & 3.6 & 3.7 \\
0.2 & $3.624 \times 10^{-6}$ & $2.396 \times 10^{-8}$ & $1.008 \times 10^{-9}$ & 7.2 & 5.9 \\
0.3 & $1.387 \times 10^{-5}$ & $1.768 \times 10^{-8}$ & $2.106 \times 10^{-9}$ & 9.6 & 6.3 \\
0.4 & $5.279 \times 10^{-5}$ & $8.602 \times 10^{-7}$ & $6.609 \times 10^{-10}$ & 5.9 & 8.1 \\
0.5 & $4.043 \times 10^{-4}$ & $2.555 \times 10^{-5}$ & $1.637 \times 10^{-6}$ & 3.9 & 3.9 \\
\hline
\end{tabular}

Table 12. The absolute errors of the fourth order derivatives of $y_{2}$ at the knots and the numerical convergence orders.

\begin{tabular}{cccccc}
\hline$x_{\boldsymbol{i}}$ & $\boldsymbol{E}_{\mathbf{4}}\left(\boldsymbol{x}_{\boldsymbol{i}}, \mathbf{1 0}\right)$ & $\boldsymbol{E}_{\mathbf{4}}\left(\boldsymbol{x}_{\boldsymbol{i}}, \mathbf{2 0}\right)$ & $\boldsymbol{E}_{\mathbf{4}}\left(\boldsymbol{x}_{\boldsymbol{i}}, \mathbf{4 0}\right)$ & $\boldsymbol{O}_{\mathbf{4}}\left(\boldsymbol{x}_{\boldsymbol{i}}, \mathbf{1 0}, \mathbf{2 0}\right)$ & $\boldsymbol{O}_{\mathbf{4}}\left(\boldsymbol{x}_{\boldsymbol{i}}, \mathbf{1 0}, \mathbf{4 0}\right)$ \\
\hline-0.5 & $5.824 \times 10^{-3}$ & $7.682 \times 10^{-4}$ & $1.098 \times 10^{-4}$ & 2.9 & 2.8 \\
-0.4 & $1.743 \times 10^{-3}$ & $7.014 \times 10^{-5}$ & $2.500 \times 10^{-5}$ & 4.6 & 3.0 \\
-0.3 & $1.364 \times 10^{-4}$ & $1.006 \times 10^{-4}$ & $1.931 \times 10^{-5}$ & 0.4 & 1.4 \\
-0.2 & $6.769 \times 10^{-5}$ & $8.285 \times 10^{-5}$ & $1.238 \times 10^{-5}$ & -0.3 & 1.2 \\
-0.1 & $3.940 \times 10^{-4}$ & $6.240 \times 10^{-5}$ & $7.654 \times 10^{-6}$ & 2.6 & 2.8 \\
0.0 & $3.315 \times 10^{-4}$ & $4.161 \times 10^{-5}$ & $2.369 \times 10^{-6}$ & 2.9 & 3.5 \\
0.1 & $2.691 \times 10^{-4}$ & $2.078 \times 10^{-5}$ & $3.074 \times 10^{-6}$ & 3.6 & 3.2 \\
0.2 & $5.980 \times 10^{-4}$ & $7.186 \times 10^{-8}$ & $8.315 \times 10^{-6}$ & 13.0 & 3.0 \\
0.3 & $5.313 \times 10^{-4}$ & $1.816 \times 10^{-5}$ & $1.326 \times 10^{-5}$ & 4.8 & 2.6 \\
0.4 & $2.448 \times 10^{-3}$ & $1.408 \times 10^{-5}$ & $1.853 \times 10^{-5}$ & 7.4 & 3.5 \\
0.5 & $5.332 \times 10^{-3}$ & $7.329 \times 10^{-4}$ & $1.119 \times 10^{-4}$ & 2.8 & 2.7 \\
\hline
\end{tabular}


Table 13. The absolute errors of the fifth order derivatives of $y_{2}$ at the knots and the numerical convergence orders.

\begin{tabular}{cccccc}
\hline$x_{\boldsymbol{i}}$ & $E_{\mathbf{5}}\left(\boldsymbol{x}_{\boldsymbol{i}}, \mathbf{1 0}\right)$ & $E_{5}\left(\boldsymbol{x}_{\boldsymbol{i}}, \mathbf{2 0}\right)$ & $E_{5}\left(\boldsymbol{x}_{\boldsymbol{i}}, \mathbf{4 0}\right)$ & $\boldsymbol{O}_{\mathbf{5}}\left(\boldsymbol{x}_{\boldsymbol{i}}, \mathbf{1 0}, \mathbf{2 0}\right)$ & $\boldsymbol{O}_{\mathbf{5}}\left(\boldsymbol{x}_{\boldsymbol{i}}, \mathbf{1 0}, \mathbf{4 0}\right)$ \\
\hline-0.4 & $2.797 \times 10^{-2}$ & $2.832 \times 10^{-3}$ & $1.036 \times 10^{-3}$ & 3.3 & 2.3 \\
-0.3 & $7.801 \times 10^{-3}$ & $2.489 \times 10^{-4}$ & $2.683 \times 10^{-4}$ & 4.9 & 2.4 \\
-0.2 & $2.854 \times 10^{-3}$ & $1.320 \times 10^{-4}$ & $7.551 \times 10^{-6}$ & 4.4 & 4.2 \\
-0.1 & $3.345 \times 10^{-4}$ & $2.027 \times 10^{-4}$ & $3.936 \times 10^{-5}$ & 0.7 & 1.5 \\
0.0 & $1.042 \times 10^{-3}$ & $2.083 \times 10^{-4}$ & $5.694 \times 10^{-5}$ & 2.3 & 2.0 \\
0.1 & $3.340 \times 10^{-4}$ & $2.081 \times 10^{-4}$ & $5.359 \times 10^{-5}$ & 0.7 & 1.3 \\
0.2 & $2.978 \times 10^{-3}$ & $2.027 \times 10^{-4}$ & $4.824 \times 10^{-5}$ & 3.8 & 2.9 \\
0.3 & $7.583 \times 10^{-3}$ & $9.012 \times 10^{-5}$ & $5.461 \times 10^{-5}$ & 6.3 & 3.5 \\
0.4 & $2.765 \times 10^{-2}$ & $1.876 \times 10^{-3}$ & $2.563 \times 10^{-5}$ & 3.8 & 5.0 \\
\hline
\end{tabular}

Moreover, all of the absolute errors in these tables are very satisfactory and well accepted. Making a further observation on these tables, we find that the errors at the inner knots are much better than the errors at the left endpoint and the right endpoint. The numerical phenomenon is natural and reasonable, because we only make use of $n$ integral values (2) and do not make use of any exact end conditions. It shows that the influence of the artificial end conditions on the inner errors is limited. In fact, the inner approximation errors are mainly determined by the given $n$ integral values in (2), while the boundary errors are mainly effected by the artificial end conditions. It is checked that our inner errors of $y_{1}$ in Tables 2-6 are similar to the ones in $[2,14,18]$, which are obtained by using five or seven additional exact end conditions. It shows that our new method can obtain satisfactory approximation results by using fewer data than the methods in $[2,14,18]$. The performance is very encouraging.

Finally, we give some discussion on fifth order derivative approximation. We remark that we use:

$$
W_{i}=\frac{s^{(4)}\left(x_{i+1}\right)-s^{(4)}\left(x_{i-1}\right)}{2 h}=\frac{F_{i+1}-F_{i-1}}{2 h}
$$

to approximate $y^{(5)}\left(x_{i}\right)$ in this paper, $i=1,2, \ldots, n-1$. See Tables 7 and 13 for our numerical results of the fifth order derivatives. Take $y_{1}$ as a comparison example. See Table 14 for the comparison of the maximum absolute errors of the fifth order derivatives $y_{1}^{(5)}\left(x_{i}\right)$ obtained by our current method and the methods in $[18,19]$. Obviously, our results are very accurate and surprising because they are obtained by only using the integral values (2) with no exact end conditions, while the results of [18] are obtained by using the integral values (2) and five additional exact end conditions $\left(y\left(x_{0}\right), y^{\prime}\left(x_{1}\right), y^{\prime}\left(x_{n-1}\right), y^{\prime \prime \prime}\left(x_{1}\right)\right.$ and $\left.y^{\prime \prime \prime}\left(x_{n-1}\right)\right)$, as well. Hence, our approximation method for the fifth order derivatives at the inner knots is more preferable.

Table 14. Comparison of the maximum absolute errors of the fifth order derivatives of $y_{1}$.

\begin{tabular}{cccc}
\hline & $\boldsymbol{n}=\mathbf{1 0}$ & $\boldsymbol{n}=\mathbf{2 0}$ & $\boldsymbol{n}=\mathbf{4 0}$ \\
\hline Current Method & $5.871 \times 10^{-2}$ & $1.752 \times 10^{-2}$ & $5.021 \times 10^{-3}$ \\
{$[18]$} & $1.365 \times 10^{-1}$ & $6.794 \times 10^{-2}$ & $3.427 \times 10^{-2}$ \\
{$[19]$} & $3.15 \times 10^{-1}$ & $1.49 \times 10^{-1}$ & $7.15 \times 10^{-2}$ \\
\hline
\end{tabular}

\section{Conclusions}

In this paper, an effort that is different from the ones in [1,2,13-19] is made to construct a new kind of integro quintic spline without exact end conditions. The demands of exact end conditions in many old methods, such as $[1,2,14,15,18]$, for integro interpolation have been relaxed and deleted in the new method. The good feature makes the current method possess wider applications than many other methods. Moreover, the method is easy to apply, and the obtained integro quintic spline 
has satisfactory approximation abilities in approximating a function and its first order to fifth order derivatives. Hence, the new method is very effective for integro interpolation.

Acknowledgments: This work was supported by the National Natural Science Foundation of China (Grant No. 11501533). The author appreciates the reviewers and the editors very much for their careful reading, professional review and valuable suggestions.

Conflicts of Interest: The author declares no conflict of interest.

\section{References}

1. Behforooz, H. Approximation by integro cubic splines. Appl. Math. Comput. 2006, 175, 8-15.

2. Behforooz, H. Interpolation by integro quintic splines. Appl. Math. Comput. 2010, 216, 364-367.

3. Boneva, L.I.; Kendall, D.G.; Stefanov, I. Spline transformations: three new diagnostic aids for the statistical data-analyst. J. R. Stat. Soc. Ser. B 1971, 33, 1-70.

4. Boujraf, A.; Sbibih, D.; Tahrichi, M.; Tijini, A. A simple method for constructing integro spline quasi-interpolants. Math. Comput. Simul. 2015, 111, 36-47.

5. Costantini, P.; Pelosi, F. Shape preserving histogram approximation. Adv. Comput. Math. 2007, 26, 205-230.

6. DeBoor, C. A Practical Guide to Splines; Springer: New York, NY, USA, 1978.

7. Delhez, E.J.M. A spline interpolation technique that preserve mass budget. Appl. Math. Lett. 2003, 16, 17-26.

8. Demichelis, V. Piecewise weighted mean functions and histograms. Rocky Mt. J. Math. 1998, 28, 1259-1265.

9. Epstein, E.S. On obtaining daily climatological values from monthly means. J. Clim. 1991, 4, 365-372.

10. Huang, J.; Chen, Y. A regularization method for the function reconstruction from approximate average fluxes. Inverse Probl. 2005, 21, 1667-1684.

11. Killworth, P. Time interpolation of forcing fields in ocean models. J. Phys. Oceanogr. 1996, 26, 136-143.

12. Klimchuk, J.A.; Patsourakos, S.; Tripathi, D. Intensity conserving spectral fitting. Sol. Phys. 2016, 291, 55-65.

13. Lang, F.G.; Xu, X.P. On integro quartic spline interpolation. J. Comput. Appl. Math. 2012, 236, 4214-4226.

14. Lang, F.G.; Xu, X.P. Quintic B-spline method for integro interpolation. Appl. Math. Comput. 2015, 263, 353-360.

15. Wu, J.; Zhang, X. Integro sextic spline interpolation and its super convergence. Appl. Math. Comput. 2013, 219, 6431-6436.

16. Wu, J.; Zhang, X. Integro quadratic spline interpolation. Appl. Math. Model. 2015, 39, 2973-2980.

17. Xu, X.P.; Lang, F.G. Quintic B-spline method for function reconstruction from integral values of successive subintervals. Numer. Algor. 2014, 66, 223-240.

18. Zhanlav, T.; Mijiddorj, R. Integro quintic splines and their approximation properties. Appl. Math. Comput. 2014, 231, 536-543.

19. Zhanlav, T.; Mijiddorj, R.; Behforooz, H. Construction of local integro quintic splines. Commun. Numer. Anal. 2016, 2016, 167-179.

20. Schoenberg, I.J. Contribution to the problem of approximation of equidistant data by analytic functions. Q. Appl. Math. 1946, 4, 45-99; 112-141.

21. Wang, R.H. Numerical Approximation; Higher Education Press: Beijing, China, 1999.

(c) 2017 by the author. Licensee MDPI, Basel, Switzerland. This article is an open access article distributed under the terms and conditions of the Creative Commons Attribution (CC BY) license (http://creativecommons.org/licenses/by/4.0/). 